\title{
Corrigendum
}

\section{The minimum crossing number of essential tangles}

[J. Knot Theory Ramifications, Vol. 23, No. 10 (2014) Article ID: 1450054]

\author{
João Miguel Nogueira* and António Salgueiro ${ }^{\dagger}$ \\ Departmento de Matemática da Universidade de Coimbra, \\ Apartado 3008, 3001-454 Coimbra, Portugal \\ *nogueira@mat.uc.pt \\ †ams@mat.uc.pt
}

Received 9 August 2016

Accepted 21 February 2017

Published 22 March 2017

\begin{abstract}
We correct some of the arguments proving that the tangles on Theorems 1.1 and 1.2 are essential.

Keywords: Essential tangles; crossing number; tangle classification.

Mathematics Subject Classification 2010: 57M25, 57M27
\end{abstract}

\section{Introduction}

In this note, we correct three arguments on the proofs of the tangles of Theorems 1.1 and 1.2 being essential. The results of the original paper remain unaltered, as stated below.

Theorem 1.1. Let $\mathcal{T}$ be a n-string essential tangle. Then

$$
c(\mathcal{T}) \geq 2 n+1
$$

We have $c(\mathcal{T})=2 n+1$ if and only if $\mathcal{T}$ is equivalent to the tangle in Fig. 1 .

Theorem 1.2. Let $\mathcal{T}$ be a n-string essential tangle, with all strings unknotted. Then

$$
c(\mathcal{T}) \geq 2 n+2 .
$$

We have $c(\mathcal{T})=2 n+2$ if and only if $T$ is equivalent to one of the tangles in Fig. 2 .

${ }^{*}$ Corresponding author. 


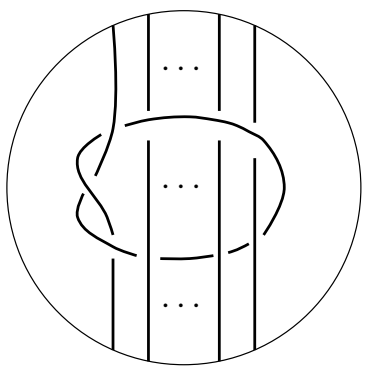

Fig. 1. The $n$-string essential tangle with the minimum crossing number.

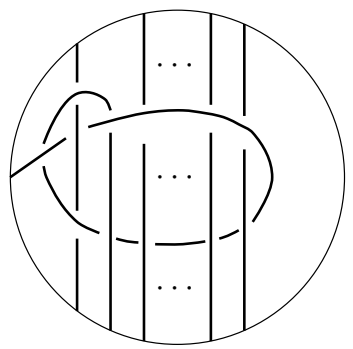

(a)

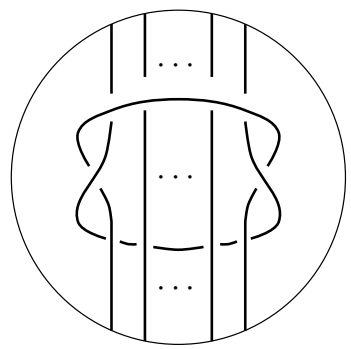

(b)

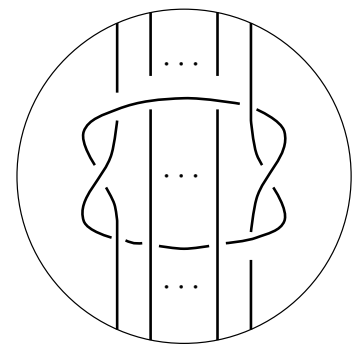

(c)

Fig. 2. The all strings unknotted $n$-string essential tangles with the minimum crossing number.

\section{The $n$-String Tangle of Theorem 1.1}

In this section, we correct the proof that the $n$-string tangle of Theorem 1.1 is essential. The original argument, on p. 1450054-7, leads to a contradiction for a trivial link having linking number $n-1$, however, the linking number of the argument can actually be $n-1$ as well as other non-zero values. When correcting this argument, we opted for another path for the proof, which is summed up in Lemma 2.2. We first give a definition of prime tangle, inspired by Bleiler [1]:

Definition 2.1. An essential tangle $(B, t)$ is said to be prime if:

(1) Any 2-sphere embedded in $B$ which meets $t$ transversely in two points is parallel to $\partial B$ or it bounds a 3 -ball in $B$ which meets $t$ in a single unknotted arc.

(2) Any properly embedded disc $D$ which meets $t$ transversely in a single point is such that $\partial D$ bounds a disc in $\partial B$ which also meets $t$ in a single point.

Note that if $(B, t)$ is a 1-string essential tangle, then it is prime if and only if the knot $t \cup \alpha$ is prime, where $\alpha$ is an arc on $\partial B$ joining the endpoints of $t$.

Lemma 2.2. Let $\mathcal{T}=\left(B, s \cup s_{n}\right)$ be a n-string tangle, where $s=s_{1} \cup \cdots \cup s_{n-1}$ and $(B, s)$ is a prime tangle. Suppose there is an arc $\alpha$ on $\partial B$ such that $s_{n} \cup \alpha$ 

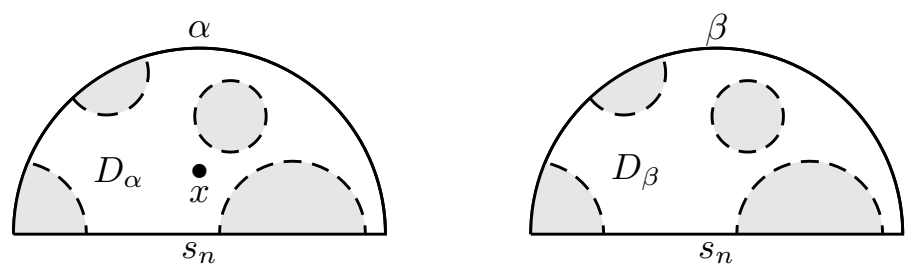

Fig. 3. The possible types of connected components $\delta$ (dashed) of $D_{\alpha} \cap D_{\beta}$ and the corresponding disks $\Delta_{\alpha}$ and $\Delta_{\beta}$ (grayed).

bounds a disk $D_{\alpha}$ that meets $s$ transversely in a single point $x$, decomposing s along $x$ into $a$ and $b$. Let $c$ be an arc on $D_{\alpha}$ from $x$ to $\partial B$. Then $\mathcal{T}$ is not essential if and only if $s$ is ambient isotopic to $a \cup c$ or $b \cup c$ in $B-s_{n}$.

Proof. Since $(B, s)$ is essential, then $\mathcal{T}$ is not essential if and only if there is a properly embedded disk in $B$ separating $s$ from $s_{n}$. It is clear that if $s$ is isotopic to $a \cup c$ or $b \cup c$, then there is such a disk, therefore $\mathcal{T}$ is not essential.

Suppose now that there is a disk separating $s$ from $s_{n}$. Since $s_{n}$ is unknotted, there is a disk $D_{\beta}$ with boundary $s_{n} \cup \beta$, where $\beta$ is a simple arc in $\partial B$ such that $D_{\beta} \cap s$ is empty.

In the following, denote the interior of $X$ by $X^{\circ}$ and the number of connected components of $X$ by $|X|$, and assume that all sub-manifolds are in general position. Suppose that $\left|D_{\alpha}^{\circ} \cap D_{\beta}^{\circ}\right|$ is minimal among all disks $D_{\beta}$ as above. In Fig. 3 we have a schematic illustration for the possible types of connected components of $D_{\alpha} \cap D_{\beta}$ in $D_{\alpha}$ and $D_{\beta}$. We will show that $\left|D_{\alpha}^{\circ} \cap D_{\beta}^{\circ}\right|=0$.

Suppose first that $D_{\alpha}^{\circ} \cap D_{\beta}^{\circ}$ contains some simple closed curve $\delta$, that bounds a disk $\Delta_{\alpha}$ in $D_{\alpha}$ and a disk $\Delta_{\beta}$ in $D_{\beta}$.

Assume $\delta$ to be innermost in $D_{\alpha}$, that is $\left|\Delta_{\alpha}^{\circ} \cap D_{\beta}^{\circ}\right|=0$. As the 2 -sphere $\Delta_{\alpha} \cup \Delta_{\beta}$ is separating in $B$, the disk $\Delta_{\alpha}$ cannot contain $x$. Hence, $\Delta_{\alpha} \cup \Delta_{\beta}$ is disjoint from $s$. By substituting $D_{\beta}^{\circ}$ by the disk $\left(D_{\beta}^{\circ}-\Delta_{\beta}\right) \cup \Delta_{\alpha}$ and perturbing it slightly, we obtain a contradiction with the minimality of $\left|D_{\alpha}^{\circ} \cap D_{\beta}^{\circ}\right|$. Therefore, $D_{\alpha}^{\circ} \cap D_{\beta}^{\circ}$ contains no simple closed curves.

Now suppose that $D_{\alpha}^{\circ} \cap D_{\beta}^{\circ}$ contains an $\operatorname{arc} \delta$, that, along with an arc of $\partial D_{\alpha}$, bounds a disk $\Delta_{\alpha}$ in $D_{\alpha}$ and, along with an arc of $\partial D_{\beta}$, bounds a disk $\Delta_{\beta}$ in $D_{\beta}$. We will assume $\delta$ to be innermost in $D_{\alpha}$, that is, $\left|\Delta_{\alpha}^{\circ} \cap D_{\beta}^{\circ}\right|=0$, and that $\Delta_{\alpha}$ doesnot contain $x$. Again, by substituting $D_{\beta}^{\circ}$ by the disk $\left(D_{\beta}^{\circ}-\Delta_{\beta}\right) \cup \Delta_{\alpha}$ and perturbing it slightly, we obtain a contradiction with the minimality of $\left|D_{\alpha}^{\circ} \cap D_{\beta}^{\circ}\right|$. Therefore, $D_{\alpha}^{\circ} \cap D_{\beta}^{\circ}$ contains no arcs, which completes the proof that $\left|D_{\alpha}^{\circ} \cap D_{\beta}^{\circ}\right|=0$.

Since the properly embedded disk $D_{\alpha} \cup D_{\beta}$ meets the prime tangle $s$ transversely in a single point, then $\alpha \cup \beta$ bounds a disk $O$ in $\partial B$ that meets $s$ in a single point. Then the sphere $D_{\alpha} \cup D_{\beta} \cup O$ meets $s$ transversely in two points. This sphere is either parallel to $\partial B$ or it bounds a 3 -ball in $B$ which meets $s$ in a single unknotted arc. In either case, $a$ or $b$ is a trivial arc in the corresponding ball separated by 

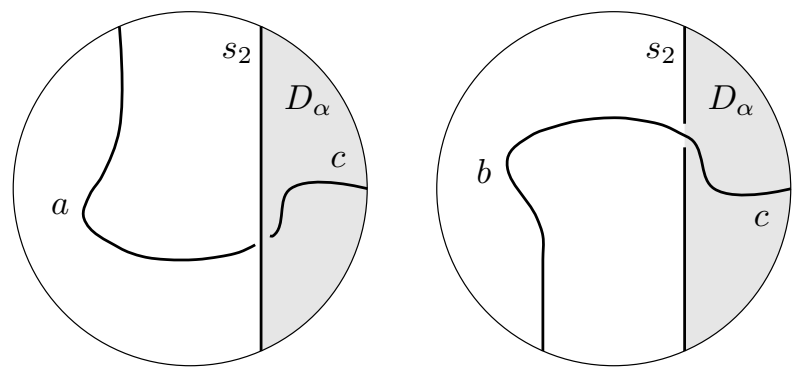

Fig. 4. If $\mathcal{T}$ is not essential, then $s_{1}$ is unknotted.

$D_{\alpha} \cup D_{\beta}$. We can then isotope this trivial arc, inside the ball containing it, into $c$. That shows that $s$ can be isotoped into $b \cup c$ or $a \cup c$, respectively.

Proof. (Proof that the $n$-string tangle of Theorem 1.1 is essential) Let $s_{1}$ be the string in $\mathcal{T}$ with the pattern of a trefoil, and $s_{2}$ be one of the remaining parallel arcs. Since the trefoil is a prime knot, then $\left(B, s_{1}\right)$ is prime. By Lemma 2.2, $\left(B, s_{1} \cup s_{2}\right)$ is essential. Otherwise, $s_{1}$ could be isotoped into one of the strings $a \cup c$ or $b \cup c$ on Fig. 4 , and $s_{1}$ would be unknotted. As all strings but $s_{1}$ are parallel to $s_{2}$, then $\mathcal{T}$ is essential.

\section{The 2-String Tangle of Fig. 2(a)}

In this section, we correct the proof that the 2-string tangle of Fig. 2(a) is essential. The original argument is on the proof of Theorem 1.2, on p. 1450054-10. In this argument, we choose to add a certain rational tangle to $\left(B, s_{1} \cup s_{2}\right)$ obtaining the knot $6_{2}$ from Rolfsen's list [3]. We then follow by assuming the tangle $\left(B, s_{1} \cup s_{2}\right)$ is inessential, hence rational, as the two strings are unknotted. From here, we isotope $s_{2}$ to the complement of $B$ and jump to the conclusion that the knot $6_{2}$ is the connected sum of two unknots. The fallacy on this argument is on the assumption

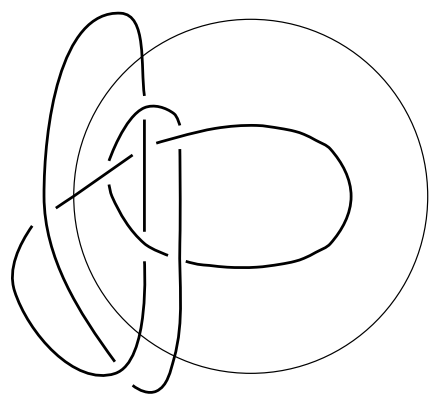

Fig. 5. A decomposition of the knot $8_{16}$ in tangles. 
that by an isotopy of $s_{2}$ to the complement of $B$, we obtain an unknotted arc. In general, the argument is not valid as it would imply that all 2-bridge knots are trivial, which is not true.

Proof. (Proof that the 2-string tangle of Fig. 2(a) is essential) The knot 816 , as in KnotInfo [2], has a decomposition defined by the tangle $\left(B, s_{1} \cup s_{2}\right)$ and a rational tangle, depicted in Fig. 5.

As observed before, if the tangle $\left(B, s_{1} \cup s_{2}\right)$ is inessential, then it is rational. This contradicts the knot $8_{16}$ being 3 -bridge, known from the classification of 2-bridge knots by Schubert [4]. (See KnotInfo [2].)

\section{The $n$-String Tangle of Theorem 1.2}

In this section, we correct the proof that the $n$-string tangles of Theorem 1.2 , for $n \geq 3$, are essential. The original argument, on p. 1450054-11, leads to a reduction on the crossing number of the diagram, but this is not a contradiction as the crossing number is not assumed to be minimal. Instead, it is minimal as a consequence of the corresponding diagram being essential (the subject of the proof itself) and previous results on the original paper. To prove that these tangles are essential, we follow a similar argument to the one in Sec. 2.

Proof. (Proof that the $n$-string tangles of Theorem 1.2 are essential) We prove that the $n$-string tangles of Fig. 2(a) are essential; the proofs for the $n$-string tangles of Figs. 2(b) and 2(c) follow similarly. Let $s_{1}$ and $s_{2}$ be the strings as in the previous section and $s_{3}$ one of the remaining parallel unknotted arcs. As all strings but $s_{1}$ and $s_{2}$ are parallel to $s_{3}$, it suffices to prove that the tangle defined by $s_{1} \cup s_{2} \cup s_{3}$ is essential. Since $\left(B, s_{1} \cup s_{2}\right)$ is essential, and both $s_{1}$ and $s_{2}$ are unknotted, then $\left(B, s_{1} \cup s_{2}\right)$ is prime. If $\left(B, s_{1} \cup s_{2} \cup s_{3}\right)$ is inessential, then, by Lemma $2.2,\left(B, s_{1} \cup s_{2}\right)$ is isotopic to one of the tangles of Fig. 6 , which is a contradiction with $\left(B, s_{1} \cup s_{2}\right)$ being essential.
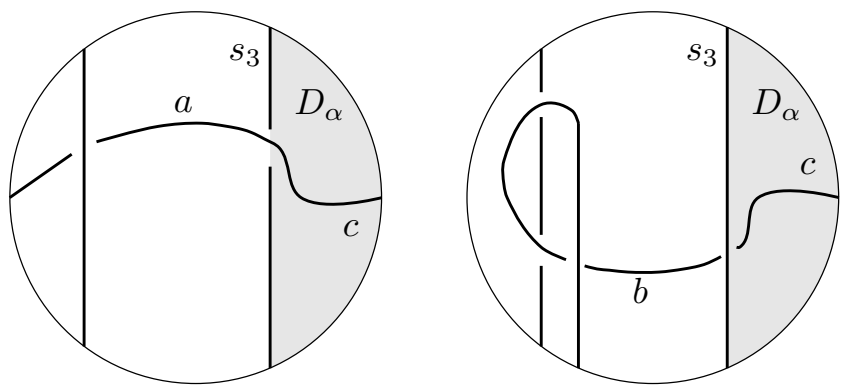

Fig. 6. If $\left(B, s_{1} \cup s_{2} \cup s_{3}\right)$ is not essential, then $\left(B, s_{1} \cup s_{2}\right)$ is not essential. 


\section{Acknowledgment}

The authors would like to thank Cameron Gordon for observing the fallacy on the argument of the original paper corrected in Sec. 3 of this corrigendum. Both authors were partially supported by the Centre for Mathematics of the University of Coimbra - UID/MAT/00324/2013, funded by the Portuguese Government through FCT/MEC and co-funded by the European Regional Development Fund through the Partnership Agreement PT2020. This work was also partially supported by the UTAustin|Portugal program CoLab on the visit of the first author to the University of Texas at Austin from April 24 to May 8 of 2016.

\section{References}

[1] S. Bleiler, Knots prime on many strings, Trans. Amer. Math. Soc. 282(1) (1984) 385-401.

[2] J. C. Cha and C. Livingston, KnotInfo: Table of knot invariants, http://www.indiana. edu/knotinfo, 2016.

[3] D. Rolfsen, Knots and Links, Vol. 346 (AMS Chelsea Publishing, 2003).

[4] H. Schubert, Knoten mit zwei Brücken, Math. Z. 65 (1956) 133-170. 